\title{
A disposable sticky electrode for the detection of commercial silver NPs in seawater
}

ARTICLE in NANOTECHNOLOGY·NOVEMBER 2013

Impact Factor: $3.67 \cdot$ DOI: 10.1088/0957-4484/24/50/505501 · Source: PubMed

CITATIONS

4
DOWNLOADS

7
VIEWS

88

5 AUTHORS, INCLUDING:

Kristina Tschulik

University of Oxford

80 PUBLICATIONS 311 CITATIONS

SEE PROFILE
Jay Thomas Cullen

University of Victoria

45 PUBLICATIONS 933 CITATIONS

SEE PROFILE 


\title{
A disposable sticky electrode for the detection of commercial silver NPs in seawater
}

\author{
W Cheng ${ }^{1}$, E J E Stuart ${ }^{1}$, K Tschulik ${ }^{1}$, J T Cullen ${ }^{2}$, R G Compton ${ }^{1}$
}

1. Department of Chemistry, Physical \& Theoretical Chemistry Laboratory, Oxford University, South Parks Road, Oxford, OX1 3QZ, United Kingdom

2. School of Earth and Ocean Sciences, University of Victoria, Victoria, BC, Canada V8W 3V6

Corresponding email: richard.compton@chem.ox.ac.uk

\begin{abstract}
:
The ability to perform efficient and affordable field detection and quantification of nanoparticles in aquatic environmental systems remains a significant technical challenge. Recently we reported a proof of concept of using "sticky" electrodes for the detection of silver nanoparticles [1]. Now a disposable electrode for detection and quantification of commercial Ag nanoparticles in natural seawater is presented. A disposable screen printed electrode is modified with cysteine and characterised by sticking and stripping experiments, with silver nanoparticle immobilisation on the electrode surface and subsequent oxidative stripping, yielding a quantative determination of the amount of Ag nanoparticles adhering to the electrode surface. The modified electrode was applied to natural seawater to mimic field-based environmental monitoring of Ag NPs present in seawater. The results demonstrated that commercial Ag NPs in natural seawater can be immobilised, enriched and quantified within short time period using the disposable electrodes without any need for elaborate experiments.
\end{abstract}

Keywords: Silver nanoparticle, disposable electrode, nanoparticle detection and quantification

\section{Introduction}

Silver nanoparticle based commercial products are available in the market from clothing to mobile phones and refrigerators, and also as an antibacterial product, they are widely used in health industry, for example in toothpaste and medical instruments. Concerns have been raised regarding possible toxic outcomes for both humans and environmental systems [2], particularly resulting from their massive inevitable release into the environment [3]. The ability to assess the quantities of commercial silver nanoparticles and also the impact of their release into the environment requires an efficient and affordable technique for nanoparticle detection and quantification. However, both the extremely low concentration of nanoparticles present in aquatic environments and the complex matrix presented by environmental waters 
make the reliable detection and quantification of nanoparticles in aquatic environmental systems a significant challenge [4].

Electrochemical methods, as a alternative method to optical methods generally requiring high cost and complex optics, provides a more efficient and cost-effective approach for detection of nanoparticles (NPs) [5-11]. Toward this goal, our group has developed a novel nanoimpact method based on the detection of NPs upon their collision with the imposed electrode. This method has been successfully applied for determining the size [5], concentration [6], molecular tag [7] and aggregation [8] of silver nanoparticles, including in real environmental media [9]. However, a limitation of this method is that it requires the collection, transport and then analysis of samples in separate media which may compromise the concentration and original aggregation state of the NPs.

Most recently, we have proposed a novel detection approach towards Ag NPs by using a modified glass carbon electrode which involves immersing such a "sticky" electrode into the medium of interest for a certain period allowing nanoparticles to impact and adsorb on the electrode and analysing the amount of NPs immobilised on the electrode surface e.g. by anodic stripping voltammetry [1]. A cost-efficient, disposable, reliable method for future infield sensing devices is of great desire. Following the proof of concept of using this "sticky" electrode method, here we report the use of a disposable sticky electrode for the detection of commercial silver NPs in seawater. In this report, a disposable screen printed disposable electrode is modified and characterised by consequent sticking and stripping experiments. The disposable electrode is "dropped" into environmental seawater containing silver NPs for a short period of time without any further treatment and silver NPs in seawater immobilised to the electrode were detected and quantified in real-time. This is the first time commercially available nanoparticles suspended (to mimic "release") in environmental seawater have been detected and quantified using a cost-efficient disposable electrode. We believe this simple and efficient approach opens up a new door to low-cost, real-time field detection of silver NPs in natural aquatic environments, which has significant commercial and applied environmental research implications. 


\section{Experimental}

\subsection{Silver Nanoparticles}

Commercial colloidal silver nanoparticles (Higher Nature) were purchased from OneClickPharmacy (oneclickpharmacy.com). The product label states that the spray contains high stability active colloidal silver with a small particle size (0.0006 to 0.005 microns) at 10 parts per million. Sodium perchlorate and sodium chloride were acquired from Aldrich and all solutions were made up using ultrapure water of resistivity $\sim 18.2 \mathrm{M} \Omega \mathrm{cm}$ (Millipore) at $25^{\circ} \mathrm{C}$.

In-house silver nanoparticles are synthesised by adding trisodium citrate (BDH Laboratory) into boiling silver nitrate, where citrate acted as both the reducing and capping agent, as described in [12]. According to [12], the synthesised nanoparticles were found to be spherical in shape and the size was determined to be about $27 \mathrm{~nm}$ in diameter. By relating the size to the overall concentration of $\mathrm{Ag}(1 \mathrm{mM})$, the concentration of $\mathrm{Ag}$ NPs in the stock suspension was calculated to be about $1.73 \times 10^{-9} \mathrm{~mol} / \mathrm{L}$.

\subsection{Seawater Sampling}

Seawater samples were collected from Saanich Inlet, an anoxic fjord in British Columbia, Canada. Samples were collected by fixing 5 L Niskin bottles (General Oceanics, Miami, FL USA) to a stainless steel hydrowire and then tripping the bottles with Teflon coated messengers. Once seawater samples had been collected using the Niskin bottles, they were directly transferred into $250 \mathrm{~mL}$ low density polyethylene bottles that were trace metal clean and stored at $4^{\circ} \mathrm{C}$ for later use in the laboratory.

\subsection{Electrochemical experiments}

All electrochemical experiments were performed at room temperature in a conventional three electrode setup using an Autolab II potentiostat (Metrohm-Autolab BV, Netherlands). Silver screen printed electrodes were manufactured by Dropsens S. L. (Llanera, Spain) and obtained from Metrohm Autolab (Runcorn, Cheshire, UK). They consist of a $4.0 \mathrm{~mm}$ diameter carbon working electrode, a silver pseudo reference electrode and a carbon counter electrode 
(Figure 1). The electrochemical experiments are referenced to the integrated silver pseudo reference electrode throughout this work unless stated otherwise.

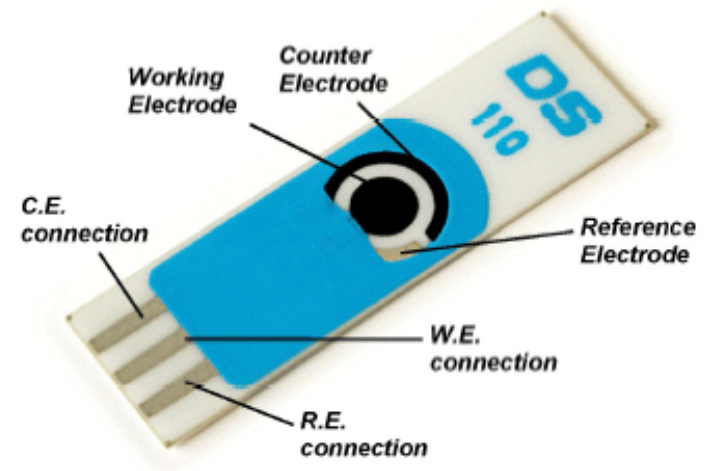

Figure 1 Schematic of Dropsens screen printed electrode

\subsection{Modifying screen printed electrodes}

The screen printed electrodes was rinsed with ethanol and water and dried with a stream of nitrogen gas. The screen printed carbon working electrodes were modified by electrochemically depositing L-cysteine using cyclic voltammetry in a solution of $5 \mathrm{mM} \mathrm{L}$ cysteine $\left(\mathrm{C}_{3} \mathrm{H}_{7} \mathrm{NO}_{2} \mathrm{~S}\right.$, Lancaster) in a PBS buffer $(\mathrm{pH}=7)$. L-cysteine solution was freshly prepared immediately prior to use. The experiment was conducted under a nitrogen atmosphere after the solution was purged thoroughly with nitrogen gas. Five scans were performed from 0 to $1.5 \mathrm{~V}$ versus the integrated silver pseudo reference electrode at a scan rate of $20 \mathrm{mV} / \mathrm{s}$. After the scans, the electrodes were rinsed with ethanol and water again to remove physically adsorbed L-cysteine on the surface. The modified electrodes are stored at room temperature and ready to use.

\subsection{Silver nanoparticle sticking}

In situ immobilisation and thus enrichment of these nanoparticles on an electrode was achieved by nanoparticle sticking experiments to determine the concentration of Ag NPs in a sample solution. After modifying the electrode, the screen printed electrode pattern was 
immersed in a suspension of either in-house made or commercially available AgNPs suspension for a certain period of time (0-960 min) and the subsequent oxidative stripping (see "silver nanoparticle stripping") was employed to quantify the amount of adherent AgNPs. A $0.5 \mathrm{~mL}$ AgNPs stock suspension in $15 \mathrm{~mL}$ water (an overall silver concentration of about $33 \mu \mathrm{M}, \mathrm{Ag}$ NPs concentration of $5.3 \times 10^{-11} \mathrm{~mol} / \mathrm{L}$ ) was used for sticking experiments of in-house Ag NPs. In-house Ag NPs suspension was diluted to an overall silver concentration of about $3.3 \mu \mathrm{M}, \mathrm{Ag}$ NPs concentration of $5.3 \times 10^{-12} \mathrm{~mol} / \mathrm{L}$. For sticking experiments of commercial Ag NPs, $6 \mathrm{~mL}$ Ag NPs commercial stock suspension was added to $12 \mathrm{~mL}$ seawater giving an overall silver concentration of $3.3 \mathrm{ppm}$. Considering that the commercial Ag NPs were characterised to have a radius of about $10 \mathrm{~nm}$ [13], the concentration of the commercial Ag NP suspension was about $1.3 \times 10^{-10} \mathrm{~mol} / \mathrm{L}$ in seawater.

\subsection{Silver nanoparticle stripping}

Following sticking the screen printed electrode for a certain period, the electrode was transferred for stripping to a freshly prepared aqueous solution of a $0.1 \mathrm{M} \mathrm{NaClO}_{4}$, while in the experiment of mimicking field-based environmental monitoring of Ag NPs present in seawater, the electrode was not transferred but remained in seawater for the subsequent stripping. Linear sweep voltammetry (LSV) was performed by scanning the potential linearly from 0-0.6 V versus an integrated silver pseudo reference electrode at a scan rate of $20 \mathrm{mV} / \mathrm{s}$. The potential region was chosen since this region was found to be suitable for quantitative oxidation of Ag NPs in $0.1 \mathrm{M} \mathrm{NaClO}_{4}[1]$ and also in the presence of chloride ions typical of seawater media [13].

\subsection{Detection of commercial silver nanoparticle in environmental seawater}

In order to demonstrate the applicability of this disposable "sticky" electrode to real-time detection and quantification of Ag NPs present in environmental seawater, the detection of commercial silver nanoparticle was performed in natural seawater. The commercial Ag NPs stock was suspended in seawater to achieve a final concentration of silver colloidal nanoparticles of $3.3 \mathrm{ppm}$. The electrode was then "dropped" in this Ag NPs containing seawater for various sticking times between 0 and $60 \mathrm{~min}$. Following sticking, the electrode 
was subsequently scanned in the exact same seawater through linear sweep voltammetry as described above, to determine the amount of Ag NPs adhering to the electrode.

\section{Results and discussion}

\subsection{Surface modification by electrochemical deposition of L-cysteine}

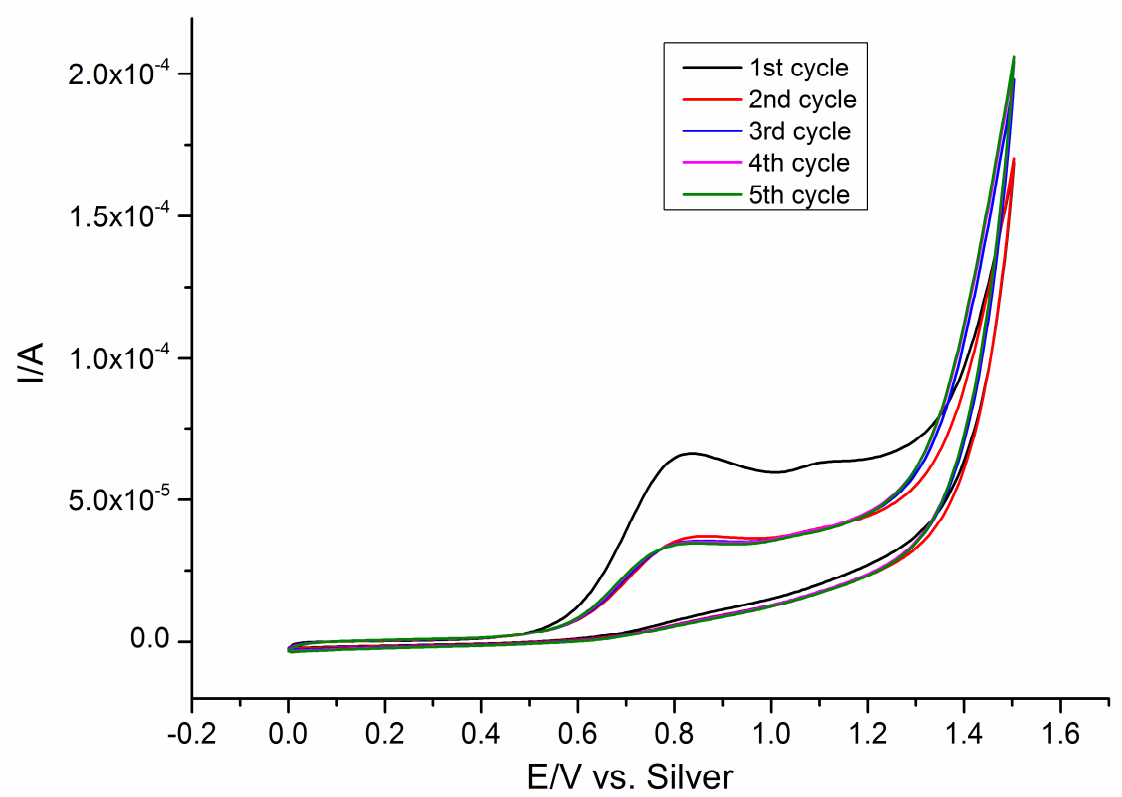

Figure 2 Cyclic voltammograms recorded in PBS buffer $(\mathrm{pH}=7)$ containing $5 \mathrm{mM}$ cysteine, initial potential , $0.0 \mathrm{~V}$ (vs. silver pseudo reference electrode), scan rate: $20 \mathrm{mV} / \mathrm{s}$

Figure 2 shows anodic immobilisation of L-cysteine on the screen printed carbon electrode by cyclic voltammetry. The film was grown on the electrode by 5 cycles of voltammetric scans between 0 and $1.5 \mathrm{~V}$. An oxidation peak was observed at about $0.85 \mathrm{~V}$ in the first anodic scan, and no cathodic peak was observed on the reverse scan within the investigated potential range. This is expected, as it is known that the electro-oxidation of L-cysteine is a chemically irreversible process [14].

The consecutive scans show that anodic peak current decreased after the first scan. This observation can be attributed to the passivation of the electrode, related to the grafting of L- 
cysteine or produced cystine absorbed on the surface of electrode. Mechanisms of immobilisation of L-cysteine proposed in the literature assumes the oxidation of the amimo group of cysteine generating cation radicals and subsequent carbon-nitrogen linkages on some carbon materials such as glassy carbon electrode [15].

\subsection{Characterising the modified electrode by sticking and stripping synthesised in-house Ag NPs}

To characterise the L-cysteine-modified screen printed carbon electrode, Ag NPs sticking experiments were performed by immersing the modified electrode in a suspension of synthesised in-house Ag NPs (53 pM) for various sticking times, for which the resulting oxidation curves of stripping by LSV are shown in Figure 3(a). The fact that no oxidative stripping peaks were observed for sticking time below $60 \mathrm{~min}$ for the unmodified electrode (data not shown) in the same condition suggests that the "sticky" performance of the Lcysteine modified electrode was dramatically improved due to the high affinity of L-cysteine functional groups (such as sulfide) for Ag. 


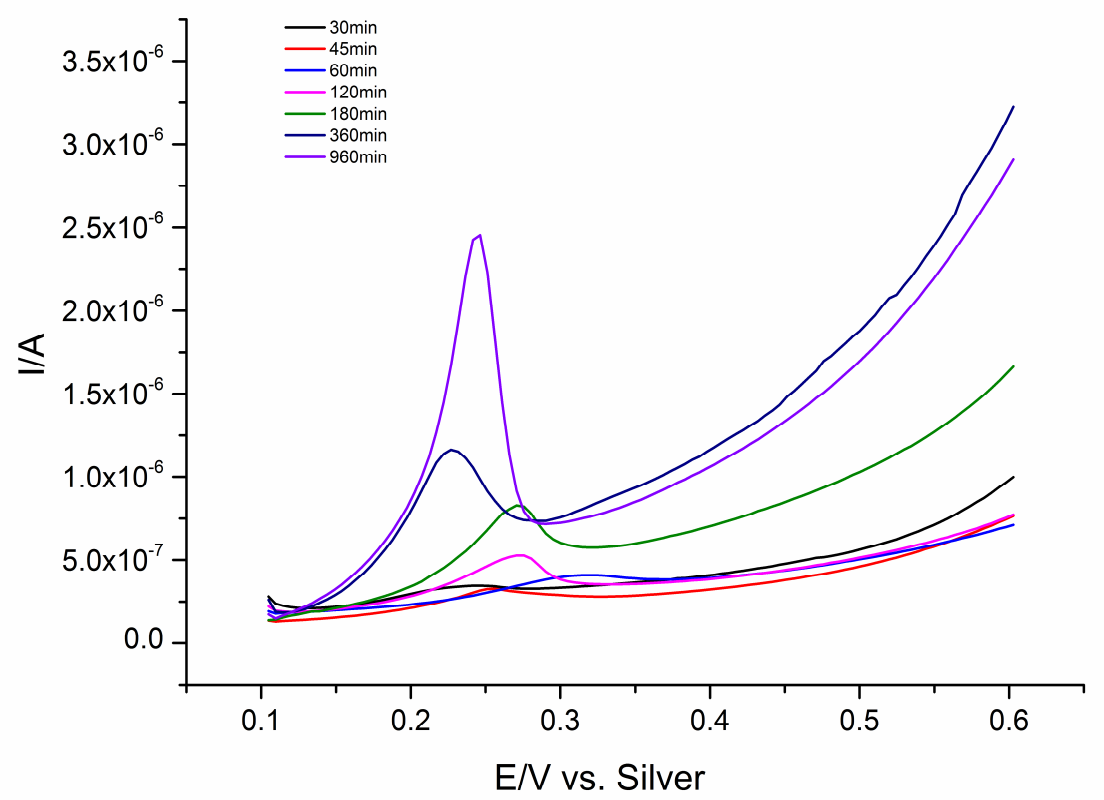

Figure 3 (a) Linear sweep voltammograms showing the oxidative stripping of Ag NPs from cysteinemodified screen printed carbon electrode in $0.1 \mathrm{M} \mathrm{NaClO}_{4}$ after immersion in $\mathrm{Ag} \mathrm{NP}$ containing water for various sticking time (min): 30 (black), 45 (red), 60 (blue), 120 (magenta), 180 (olive), 360 (navy) and violet (960).

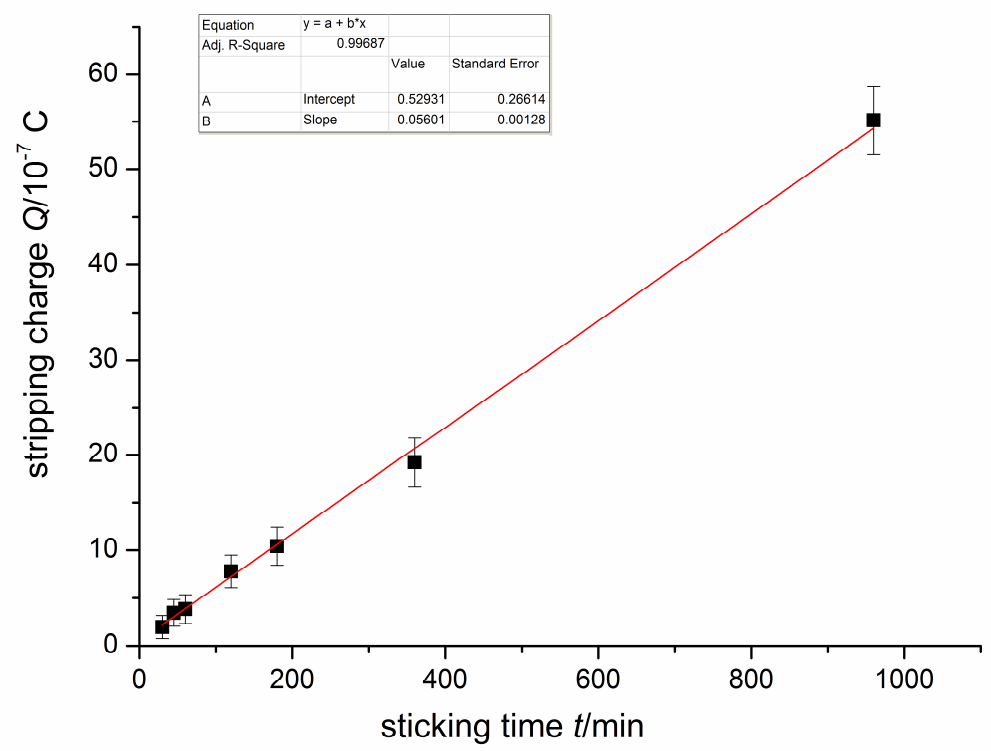

Figure 3(b) Stripping charge as function of the sticking time after sticking for a cysteine-modified screen printed carbon electrode. Mean value and standard deviation of each data point were obtained from at least two repeats. 


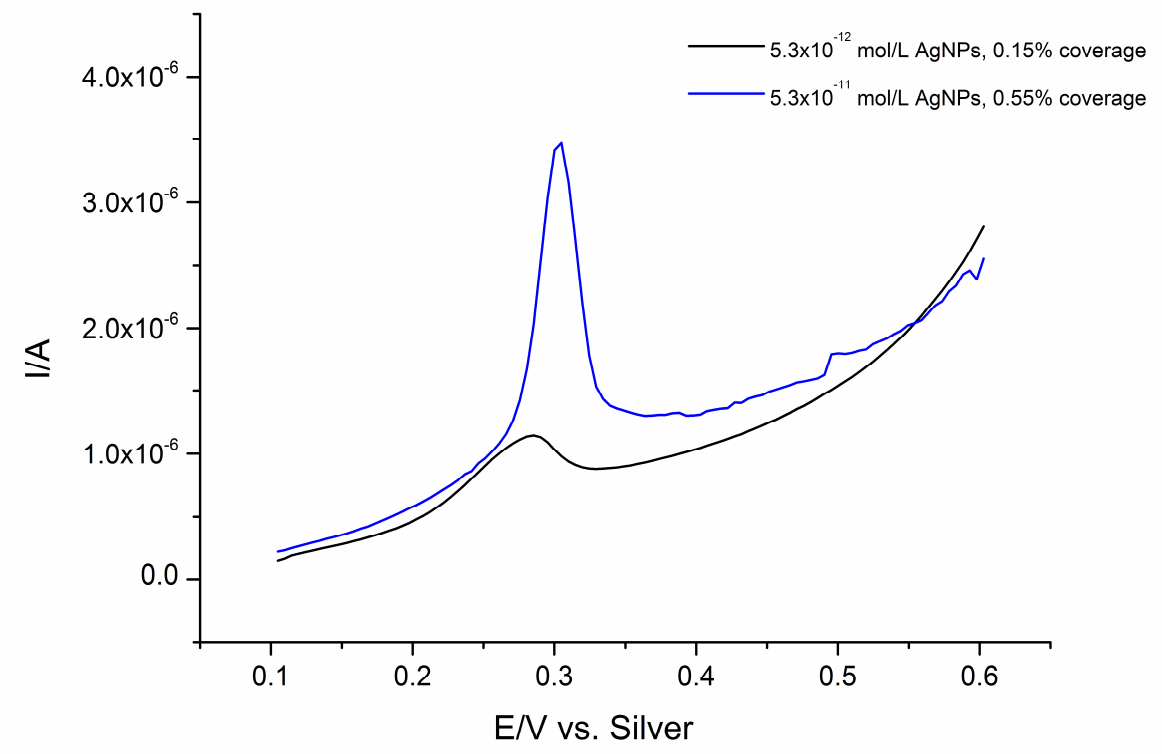

Figure 4 Linear sweep voltammograms of the oxidative stripping of Ag NPs from cysteine-modified screen printed carbon electrode in $0.1 \mathrm{M} \mathrm{NaClO}_{4}$ after sticking in water containing $\mathrm{Ag} \mathrm{NP}$ concentration of $5.3 \times 10^{-11} \mathrm{~mol} / \mathrm{L}$ (blue) and $5.3 \times 10^{-12} \mathrm{~mol} / \mathrm{L}$ (black).

Figure 3(b) shows that a linear increase of the stripping charge versus sticking time was observed for sticking times of up to $960 \mathrm{~min}$. This is interesting, in comparison to the results on the glassy carbon electrode where a linear relationship between sticking time and stripping charge was observed up to only $240 \mathrm{~min}$ [1]. The improvement of linear range may be attributed to slowing down the aggregation of Ag NPs thus maintaining the Ag NP concentration by keeping the electrode in pure water in contrast to the medium of $\mathrm{NaClO}_{4}$ electrolyte, as previously reported [1]. This may also indicate that the modified screen printed electrode may have a large surface coverage of L-cysteine immobilised on the electrode where the affinity of the suspended Ag NPs on the electrode surface is not reduced over long electrode exposure times.

By analysis of the linear fit, it was revealed that Ag NP sticking rate (number of Ag NPs per min sticking to the screen printed carbon electrode) was about $5.7 \times 10^{4} \mathrm{NP} / \mathrm{min}$, 
corresponding to about $5.6 \times 10^{-9} \mathrm{C}$ per minute of sticking time in a $53 \mathrm{pM}$ suspension of $\mathrm{Ag}$ NPs.

The oxidative charge at $360 \mathrm{~min}, 960 \mathrm{~min}$ is about $1.9 \mu \mathrm{C}, 5.5 \mu \mathrm{C}$ respectively, if considering the density and molar mass of Ag, the exchange of one electron per oxidised Ag atom, the average radius of a $\mathrm{Ag}$ nanoparticle $(\mathrm{r}=13.5 \mathrm{~nm})$ and the area of the screen printed carbon

electrode $\left(1.26 \times 10^{-5} \mathrm{~m}^{2}\right)$, which equates to a surface coverage of $0.18 \%$ and $0.53 \%$. In this case, a linear increase between the number of nanoparticles sticking to the surface with time is observed when binding sites on the electrode surface are relatively unpopulated, while a Langmuir type adsorption, and thus a non-linear dependence, can be expected as binding sites approach saturation.

Figure 4 shows the oxidative peak in a diluted (5.3 pM) suspension of Ag NPs after 960 min of sticking time. The oxidative peak is clearly observed indicating that a lower Ag NP concentration of $5.3 \mathrm{pM}$ was still detectable by the modified electrode. The surface coverage of $5.3 \mathrm{pM} \mathrm{Ag} \mathrm{NP}$ after 960 min of sticking time is about $0.15 \%$, which is approximately four times less than that in $53 \mathrm{pM}$ Ag NP $(0.53 \%)$.

\subsection{Commercial Ag NPs sticking in environmental seawater samples}

Analogous sticking and stripping experiments to detect commercial Ag NPs were carried out in authentic environmental seawater medium using the modified screen printed carbon electrode. Linear sweep voltammograms resulting from various sticking time in seawater samples containing commercial Ag NPs and subsequent stripping in $0.1 \mathrm{M} \mathrm{NaClO}_{4}$ are shown in Figure 5(a). A linear increase of the oxidative stripping charge with sticking time between 0 and 100 min was observed (see Figure 4(b)). The corresponding linear fit suggests an increase of sticking charge of about $1.39 \times 10^{-8} \mathrm{C}$ per min of sticking time, relating to about $3.5 \times 10^{6} \mathrm{NP}$ stick to the electrode surface per min. A surface coverage of between $0.06 \%$ and $0.23 \%$ for various sticking times was found. This coverage is comparable with those results 
from sticking in water, indicating that a linear increase between the number of nanoparticles sticking to the surface with time is likely for small coverage in both cases.

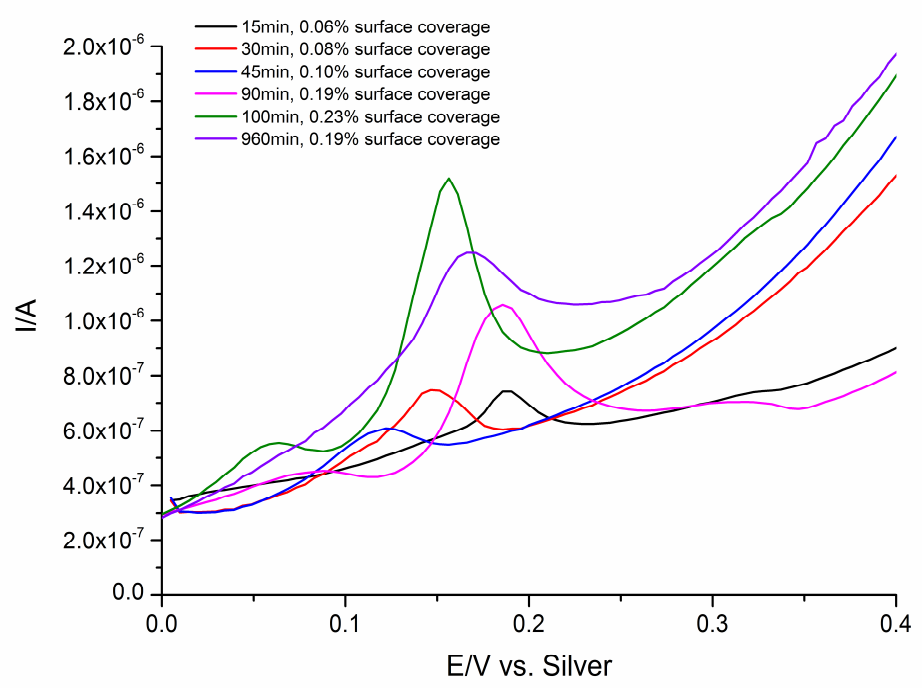

Figure 5(a)

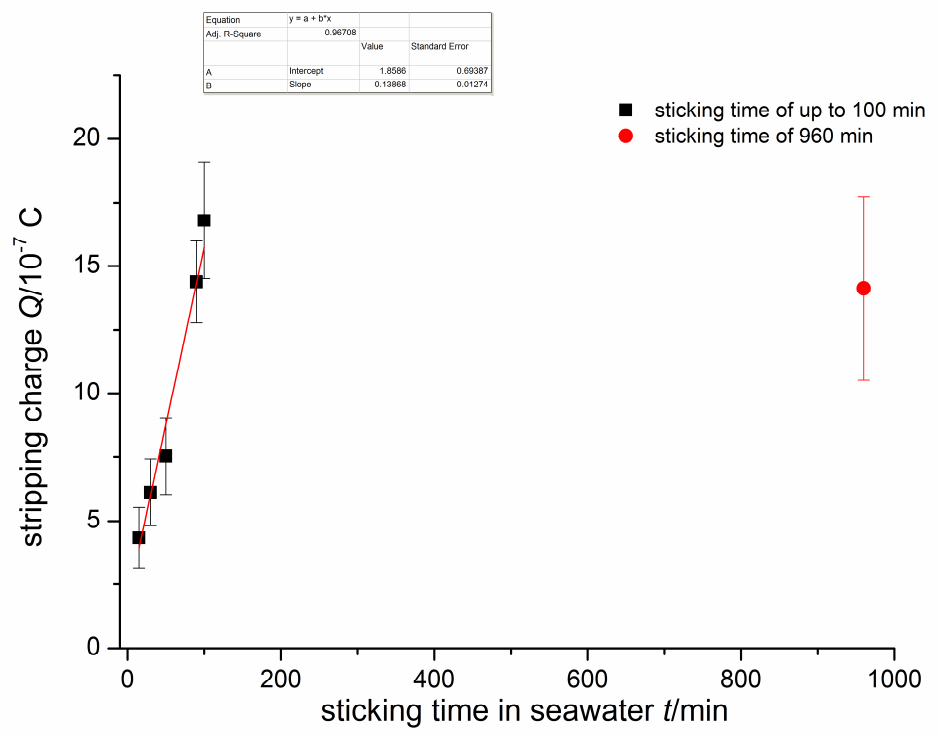

Figure 5 (b)

Figure 5 (a) Linear sweep voltammograms showing the oxidative stripping of Ag NPs from the modified screen printed carbon electrode in $0.1 \mathrm{M} \mathrm{NaClO}_{4}$ after immersion in $\mathrm{Ag} \mathrm{NP}$ containing sweater for various sticking time (min): 15 (black), 30 (red), 45 (blue), 90 (magenta), 100 (olive) and violet (960). (b) 
Stripping charge as function of the sticking time after sticking for the modified screen printed carbon electrode. Mean value and standard deviation of each data point were obtained from at least two repeats.

On some occasions another anodic peak with lower potential (at $0.06 \mathrm{~V}$ ) was observed suggesting that silver chloride was sometimes formed [13]; with the most likely source of chloride in this case being the small quantities seawater remaining (despite rinsing) on the electrode surface after sticking (also see section 3.4). An interesting observation taken from Figure 5a is the lack of an increase in stripping charge for the extended 960 min sticking time in seawater when compared with comparable times in water (see Figure 3). A possible explanation for the non-linear response in seawater over longer period of sticking might be increased Ag NP aggregation rates in higher ionic strength solutions [9] lowering the effective concentration of free Ag NPs available for sticking, with another possible contributing factor likely being degradation of the electrode due to bacterial growth or other matrix-related fouling over longer periods of immersion.

\subsection{Mimicking field-based environmental monitoring of Ag NPs present in seawater}

Considering the complex matrix in natural waters and the possible fouling of the electrode after long periods of contact with the media, the utility of the disposable electrode for efficiently monitoring of Ag NPs present in seawater with minimal exposure times was investigated.

To mimic field-based detection, commercial Ag NPs were added to seawater at a concentration of $3.3 \mathrm{ppm}$ and the modified electrode was immersed. For sticking times 0,15 , 30, 45 and $60 \mathrm{~min}$, linear sweep voltammograms were employed to quantify the amount of Ag NPs adhering to the electrode in the seawater (Figure 6(a)). 


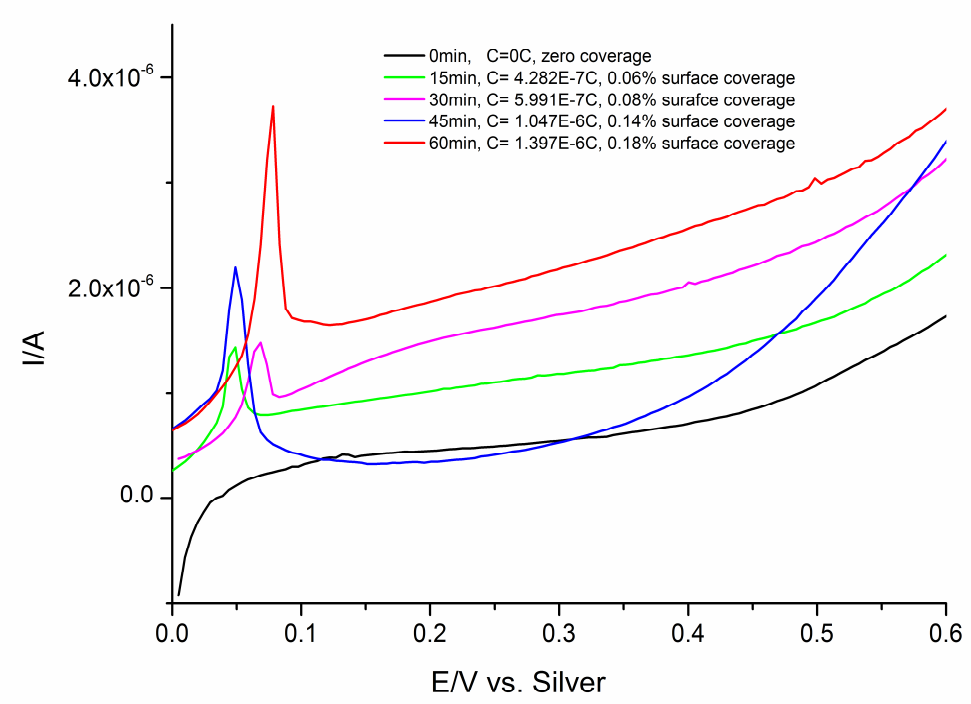

Figure 6(a)

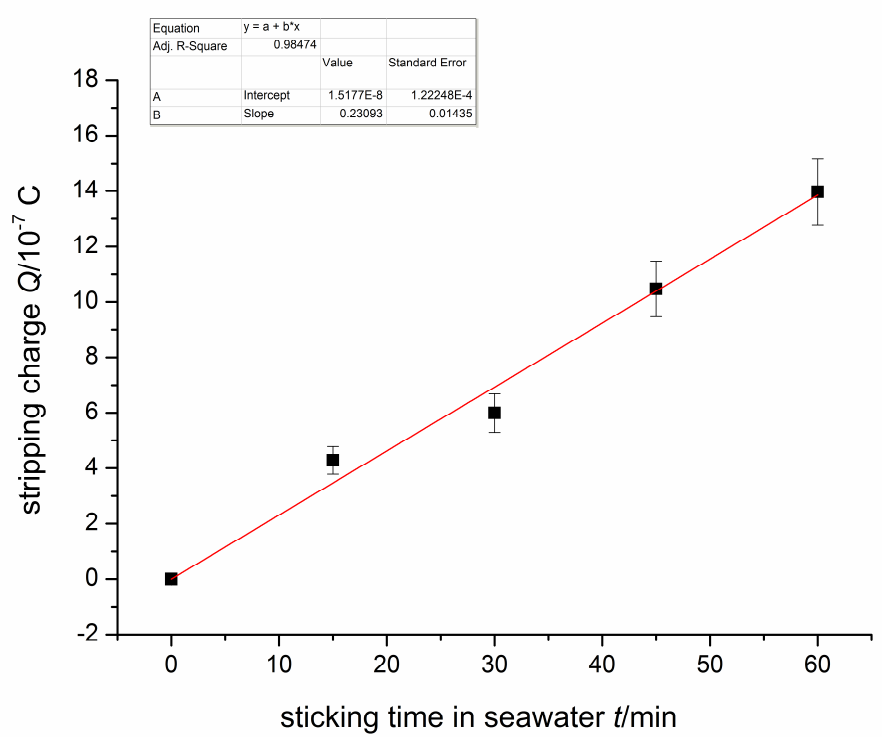

Figure 6(b)

Figure 6 (a) Linear sweep voltammograms showing the oxidative stripping of Ag NPs from the modified screen printed carbon electrode in seawater after immersion in Ag NP containing seawater for various sticking times (min): 0 (black), 15 (green), 30 (magenta), 45 (blue) and 60 (red). (b) Stripping charge as function of the sticking time in seawater for the modified screen printed carbon electrode. Mean value and standard deviation of each data point were obtained from at least two . 
The fact that no anodic peak/charge was observed for the control exposure $(0 \mathrm{~min})$, while a quantifiable anodic peak/charge was found for sticking times of 15 min or longer, suggests that the observed peaks are due to the continuous adhering of Ag NPs to the modified electrode and subsequent oxidative stripping from the electrode. While comparing the voltammetric profiles of the modified electrode in $0.1 \mathrm{M} \mathrm{NaClO}_{4}$ (Figure 4 and Figure 5) against the voltammetric stripping scan in authentic seawater media (Figure 6), it is clear that the silver nanoparticles oxidation peaks shift to a more negative potential in the seawater, which is expected from literature data on silver nanoparticle oxidation in the presence of chloride ions [16]. This additionally proves that the observed peaks resulting from sticking time of 15 min or longer are due to the adhering and stripping of Ag NPs to the modified electrode in seawater.

As before, the linear relation between sticking times and stripping charge was found (see Figure 6(b)). The number or amount of Ag NPs sticking to the electrode per minute in the

seawater was $5.7 \times 10^{6}$, given the anodic charge from the oxidative stripping of $\mathrm{Ag} \mathrm{NP}$ in seawater. Ag NP surface coverage of between zero and $0.2 \%$ for sticking times between 0 and 60 min was observed which compares well with results in water. These results demonstrate the efficacy of the modified, "sticky" electrode to immobilize, pre-concentrate and quantify Ag NPs in natural seawater.

\section{Conclusion}

In this paper, a disposable electrode for detection and quantification of commercial Ag NPs in authentic environmental seawater samples was presented. The disposable screen printed electrode was modified with cysteine and characterised by sticking and stripping experiments, which involved Ag NPs immobilisation on the electrode surface and subsequent oxidative stripping, yielding a quantative determination of the amount of Ag NPs adhering to the electrode surface. Then the modified electrode was applied to authentic environmental seawater for mimicking field-based environmental monitoring of $\mathrm{Ag}$ NPs present in seawater. The results demonstrated that Ag NPs in authentic environmental seawater can be immobilised, enriched and quantified within short time period using the modified electrode without any elaborate sample pretreatment or experimental protocol. The simplicity and efficiency with which the disposable electrode can sense Ag NPs shows high promise for in- 
field and real-time detection and quantification of Ag NPs present in natural aquatic environments, which we believe has significant commercial and environmental applications.

\section{Acknowledgments}

The Leverhulme Trust (Grant No. F/08 788/J) and the Natural Sciences and Engineering Research Council of Canada is gratefully acknowledged for funding this work.

\section{References}

[1] Tschulik K, Palgrave R G, Batchelor-McAuley C, Compton R G 2013 'Sticky electrodes' for the detection of silver nanoparticles Nanotechnology 29295502

[2] Ahamed M, AlSalhi M S and Siddiqui M K J 2010 Silver nanoparticle applications and human health Clinica Chimica Acta 411 1841-8

[3] Maynard A D et al. 2006 Safe handling of nanotechnology Nature 444 267-9

[4] Howard A G 2010 On the challenge of quantifying man-made nanoparticles in the aquatic environment J. Environ. Monit. 12 135-42

[5] Zhou Y-G, Rees N V and Compton R G 2011 Angew. Chem. Int. Ed. 50 4219-21

[6] Stuart E J E, Zhou Y G, Rees N V and Compton R G 2012 Determining unknown concentrations of nanoparticles: the particle-impact electrochemistry of nickel and silver $R S C$ Advances 2 6879-84.

[7] Zhou Y G, Rees N V, Compton R G, 2012 The electrochemical detection of tagged nanoparticles via particle-electrode collisions: nanoelectroanalysis beyond immobilisation Chemical Communications 48 2510-12.

[8] Ellison J, Tschulik K, Stuart E J E, Jurkschat K, Omanovi'c D, Uhlemann M, Crossley A and Compton R G 2013 Get more out of your data: a new approach to agglomeration and aggregation studies using nanoparticle impact experiments ChemistryOpen 2 69-75

[9] Stuart E J E, Rees N V, Cullen J T and Compton R G 2013 Direct electrochemical detection and sizing of silver nanoparticles in seawater media, Nanoscale 5 174-77

[10] Boika A, Thorgaard S N and Bard A J 2013 Monitoring the electrophoretic migration and adsorption of single insulating nanoparticles at ultramicroelectrodes J. Phys. Chem. B 117 4371-80

[11] Park J H, Thorgaard S N, Zhang B and Bard A J 2013 Single particle detection by area amplification: single wall carbon nanotube attachment to a nanoelectrode $\mathrm{J}$. Am. Chem. Soc. $1355258-61$ 
[12] Toh H S, Batchelor-McAuley C, Tschulik K, Uhlemann M, Crossley A and Compton R G 2013 The anodic stripping voltammetry of nanoparticles: electrochemical evidence for the surface agglomeration of silver nanoparticles Nanoscale 5 4884-93

[13] Stuart E J E, Tschulik K, Omanovi D, Cullen J T, Jurkschat K, Crossley A and Compton R G, 2013 Electrochemical detection of commercial silver nanoparticles: identification, sizing and detection in environmental media Nanotechnology Accepted for publication

[14] Ralph T R, Hitchman M L, Millington J P and Walsh F C 1994 The electrochemistry of Lcystine and L-cysteine: part 1: thermodynamic and kinetic studies J. Electroanal. Chem. 375 $1-15$

[15] Zhang L 2008 Covalent modification of glassy carbon electrode with cysteine for the determination of dopamine in the presence of ascorbic acid Microchim Acta 161 191-200

[16] Toh H S, Batchelor-McAuley C, Tschulik K and Compton R G 2013 Electrochemical detection of chloride levels in sweat using silver nanoparticles: a basis for the preliminary screening for cystic fibrosis Analyst 138 4292-7 